\title{
SU(3) Landau-Zener Interferometry
}

\author{
M.N. Kiselev ${ }^{1}$, K. Kikoin ${ }^{2,1}$, M.B. Kenmoe ${ }^{1,3}$ \\ 1 The Abdus Salam International Centre for Theoretical Physics, Strada Costiera 11, I-34151 Trieste, Italy \\ 2 School of Physics and Astronomy, Tel-Aviv University, Tel-Aviv 69978, Israel \\ 3 Mesoscopic and Multilayer Structures Laboratory, Faculty of Science, Department of Physics, \\ University of Dschang, Cameroon
}

PACS 73.21.La - Quantum dots

PACS 33.80. Be - Level crossing and optical pumping

PACS 03.75.Lm - Tunneling, Josephson effect, Bose-Einstein condensates in periodic potentials, solitons, vortices, and topological excitations

\begin{abstract}
We propose a universal approach to Landau-Zener problem in a three-level system. The problem is formulated in terms of Gell-Mann operators which generate $\mathrm{SU}(3)$ algebra and map the Hamiltonian on the effective anisotropic pseudospin 1 model. The vector Bloch equation for the density matrix describing the temporal evolution of three-level crossing problem is also derived and solved analytically for the case where the diabatic states of the SU(3) Hamiltonian form a triangle. This analytic solution is in excellent quantitative agreement with numerical solution of Schrödinger equation for a 3-level crossing problem. The model demonstrates oscillation patterns which radically differ from the standard patterns for two-level Landau-Zener problem. The triangle works as an interferometer and the interplay between two paths results in formation of "beats" and "steps" pattern in the time-dependent transition probability. The characteristic time scales describing the "beats" and "steps" depend on a dwell times in the triangle. These scales are related to the geometric size of interferometer. The possibilities of experimental realization of this effect in triple quantum dots and in two-well traps for cold gases are discussed.
\end{abstract}

1. Introduction. The paradigmatic problem of level crossing known as Landau-Zener model (LZM) [1- 4] is studied for eight decades (see [5] for a review). Various manifestations of LZM are found in all branches of physical sciences from astrophysics to material science. Recent progress in nanotechnology and cryogenics allow observation and application of LZM in quantum transport 6], spintronics 7], nano-magnetism 8], cold gases, including optical lattices 9], mass transport 10], quantum information processing [1]- 12, etc.

Standard approach to LZM is based on the universal $\mathrm{SU}(2)$ physics of two energy levels of the same symmetry which cross by linear variation of a control parameter (time, coordinate, energy, chemical potential, flux etc). The two states follow a diabatic basis or adiabatic (hybridized) basis under fast or slow variation of the control parameter. The probability to find a system in a given diabatic/adiabatic state at long time after passing through the crossing point is given by a simple universal one-parametric equation. Periodic (non-linear) sweep of control parameter of LZM resulting in multiple pas- sages through the crossing point allows manipulation of interference patterns by controlling the Stückelberg oscillations associated with the phases accumulated along adiabatic and non-adiabatic paths $[13$. The two-level crossing LZ theory is of paramount importance for the theory of adiabatic quantum computations [11- 12]. Recent progress in nanotechnologies opened a new possibility to use LZ interferometry for qubit manipulations 14 . The charge (Josephson) qubits are manipulated by changing gate voltage (magnetic flux) [15], [16]. The spin qubits are controlled by magnetic field 11]. Technologically, it is more convenient to manipulate qubits by electric field (gate voltages) 17.

In this paper we propose universal tools for description of 3-level LZM describing qutrits rather than qubits. Instead of Pauli matrices representing SU(2) symmetry of 2-level LZM, we use Gell-Mann matrices forming the basis for SU(3) group describing dynamical symmetry of 3-level systems. We formulate generic Hamiltonians for all possible symmetric 3-level configurations and derive the system of Bloch equations describing evolution of density matrix. 


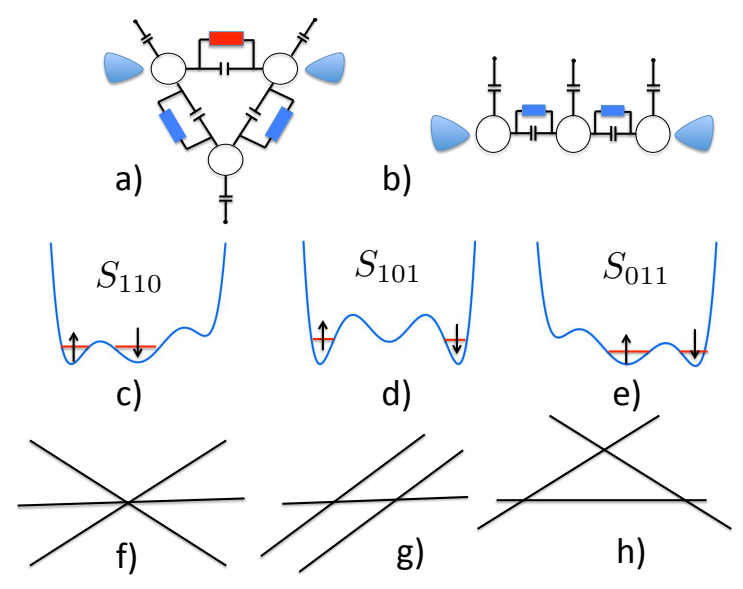

Fig. 1: (Color online) a) Triangular (TTQD) and b) Linearly arranged triple quantum dot (LTQD). Blue and red resistors control the tunneling between dots. Each dot is gated separately. c)-e) Three singlet states for TQD occupied by two electrons controlled and manipulated by the gate voltage. f)h) Three-level crossing described by Hamiltonians (2) - 4.

Numerical solution of Schrödinger equation and approximate analytical solution of Bloch equation for the density matrix of 3-level system demonstrate excellent quantitative agreement. We show that the fingerprint of 3-level LZM is a specific form of interference oscillations which differ qualitatively from those in 2-level LZM. The shape of these oscillations depends both on the geometrical size of device and on the parameter of adiabaticity.

2. Modelling three-level systems. We discuss two possible experimental realizations of three level systems: (i) spinless cold atoms in a double-well trap (DWT) 10 and (ii) triple quantum dots in a triangular (TTQD) 24 and linearly arranged (LTQD) 21] geometry.

The prototype devices for the 3-level LZM are triple quantum dots (TQD) confining two electrons in a spin singlet states [18]- 23 and double-well traps in optical lattices [24] confining two spinless cold atoms. The basic features of our model systems are illustrated in Fig. 1. Two possible quantum transport realizations of this regime are triangular [24] and linearly arranged 21] (upper panel) TQD occupied by two electrons. The three singlet states are formed by pairs of electrons $S_{110}, S_{011}$ and $S_{101}$ occupying two of three minima (middle panel). Three possible schemes of level crossing are shown in the lower panel.

Three states of doubly occupied DWT correspond to three possible occupations $(2,0),(1,1)(0,2)$ of the left and right wells. Let us fix the reference energy in the middle between the left and right levels $\varepsilon_{l, r}$, so that the tunable energy difference is $\epsilon_{l}-\epsilon_{r}=\epsilon(t)$. Having in mind the analogy between the three level system and the $S=1$ model with uniaxial anisotropy, we ascribe the pseudospin projection values $\pm 1,0$ to the states $(2,0),(1,1)(0,2)$, respectively. Then, the three crossing levels in LZ problem enumerated with accordance with this agreement are

$$
\begin{aligned}
& E_{1}=E_{2,0}=\epsilon(t)+U, \\
& E_{0}=E_{1,1}=0, \\
& E_{\overline{1}}=E_{0,2}=-\epsilon(t)+U,
\end{aligned}
$$

where $U$ is the hard-core repulsion energy of two bosons in the same well. Time evolution of these levels corresponding to a triangular configuration with $\epsilon(t)=v t$ is shown in Fig. 1 (h). The energy $U$ plays part of the parameter of easy-axis anisotropy.

Three possible configurations of the lowest state of TTQD occupied by two electrons are shown in the upper panel of Fig. 1. If the wells 1,2,3 are inequivalent, i.e. the energy levels $\epsilon_{1} \neq \epsilon_{2} \neq \epsilon_{3}$, then, each two-electron configuration is characterized by its own energy $E_{12} \neq E_{23} \neq E_{13}$. The spin state of two electrons is always singlet due to the indirect exchange via excited levels 25]. Applying in appropriate way the gate voltage $V_{g}(t)$ to corresponding dot, one may realize the level crossing. For example, changing $\epsilon_{1}(t)$ one moves the levels $E_{12}(t)$ and $E_{13}(t)$ relative $E_{23}$ thus realizing LZ regime shown in Fig. 1 (g). In case of LTQD geometry we still have three levels driven by the gate voltages, but transitions between the states $|1\rangle$ and $|\overline{1}\rangle$ are strongly suppressed like in the case of real spin 1.

When considering this system one should remember about existence of higher triplet spin states which are not immune to both external magnetic field and fluctuations of the Overhauser field associated with the hyperfine interactions. In principle these states may be involved in LZ transitions, so that the dynamical symmetry of this system will be described by $\mathrm{SO}(\mathrm{n})$ Lie groups 25 . We leave this problem for future studies.

3. General classification of three-levels crossing. Let us start with construction of equivalent spin Hamiltonian for 3-level LZ problem. For this sake we introduce the pseudospin 1 operator $\vec{S}$ and associate occupation of three crossing levels with its projections $|\overline{1}\rangle,|0\rangle, 1\rangle$. The first possibility is crossing of all three diabatic levels at one point (Fig 1,f) with effective Hamiltonian

$$
H_{1}=H_{\mathrm{LZ}}=v t S^{z}+\Delta S^{x},
$$

where $2 \Delta$ is a gap separating lower and upper adiabatic states and $v$ is the rate at which energy changes by external source in the limit $\Delta \rightarrow 0$ (we adopt the system of units $\hbar=1$ ). We refer to this model as $\mathrm{SU}(2)$ spin $S=1 \mathrm{LZ}$ model. The properties of this model are well known (see 26] for bow-tie model and 27] for $\mathrm{S}=1 \mathrm{SU}(2)$ model). The probability to remain in the same diabatic states with $S^{z}= \pm 1$ is determined by $P_{\mathrm{LZ}}=\exp (-\pi \delta / 2)$, where $\delta=\Delta^{2} / v$ is the dimensionless LZ parameter.

The second possibility is crossing of three levels at two points (Fig 1,g) with the Hamiltonian

$$
H_{2}=v t\left(S^{z}\right)^{2}+\Delta S^{x}+h S^{z} .
$$

Here $h$ denotes a tunable level splitting $h=E_{\overline{1}}-E_{1}$. The limit $h=0$ corresponds to LZ transition when two-fold 
degenerate level crosses the non-degenerate state. Note that the model is no more linear in terms of the generators of the $\mathrm{SU}(2)$ group (see [28]).

Below we concentrate on the third possibility where the three levels cross pairwise at three points forming a triangle (Fig 1,h) with the spin Hamiltonian

$$
H_{3}=v t S^{z}+\Delta S^{x}+D\left(S^{z}\right)^{2} .
$$

The last term stands for a "single-ion" easy axis anisotropy $D$. The Hamiltonian is also non-linear in terms of the $\mathrm{SU}(2)$ basis. The family of Hamiltonians $\mathrm{H}_{2}$ and $\mathrm{H}_{3}$ can be considered as a single-parametric $\mathrm{SU}(3)$ deformation of the SU(2) LZ Hamiltonian $H_{1}$. Less symmetric LZ level crossing diagrams with different velocities $v_{i}$ and tunnel rates $\Delta_{i j}$ can be considered as well. In any case the LZ Hamiltonian can be expresses via the generators of SU(3) algebra. Since the model described by $H_{3}$ is of importance for experiments in double- and triple- quantum dots 29 and contains a basic element for LZ experiments in optical lattices, we discuss below its properties leaving discussion of the model $H_{2}$ for 30 .

4. Non-adiabatic transition through a triangular interferometer. The seeming non-linearity of the model $\mathrm{H}_{3}$ is easily removed by representing it in terms of the generators of SU(3) group, namely, 8 traceless $3 \times 3$ Gell-Mann matrices 31 forming a set $\vec{\lambda}=\left\{\lambda_{\alpha}\right\}, \alpha=1 \ldots 8$. In this basis the LZ Hamiltonian casts a simple linear form describing interaction of $\vec{\Lambda}=\vec{\lambda} / 2$ with time-dependent magnetic field $\vec{B}(t)$ :

$$
H_{3}=\vec{B}(t) \cdot \vec{\Lambda}
$$

In order to minimize the number of non-zero components of $\vec{B}(t)$, it is convenient to use the rotated basis. Two versions of rotated basis are discussed below. In particular, only three combinations of $\lambda$-matrices enter the scalar product [32] in the basis of Gell-Mann matrices adjusted for a linear TQD [21], where direct transitions between the states (110) and (011) are forbidden:

$\vec{B}^{T}=\{\Delta, v t,-D / \sqrt{3}\}$,

$\vec{\Lambda}^{T}=\left\{\left(\lambda_{1}+\lambda_{6}\right) / \sqrt{2},\left(\sqrt{3} \lambda_{8}+\lambda_{3}\right) / 2,\left(-\sqrt{3} \lambda_{3}+\lambda_{8}\right) / 2\right\}$.

The numerical solution for non-adiabatic transition probabilities computed from a Schrödinger equation with the Hamiltonian $H_{3}$ is given by a blue dashed curves on Fig. 2. Both curves describe a non-adiabatic regime $\delta=\Delta^{2} / v \ll 1$. The left panel demonstrates a "beats" pattern in time dependent probability when the size of triangle is small $\eta=D^{2} / v<1$. The right panel shows a "steps" pattern when the size of triangle is large $\eta=D^{2} / v>1$. How can we understand these patterns? What are the characteristic time scales responsible for this behaviour?

Both "beats" and "steps" are attributed to the interference processes The triangle formed by three diabatic states plays a role of LZ interferometer. Schematically, the interference processes are shown in the insert 1 of the lower panel of Fig. 2. The left and upper vertices of the
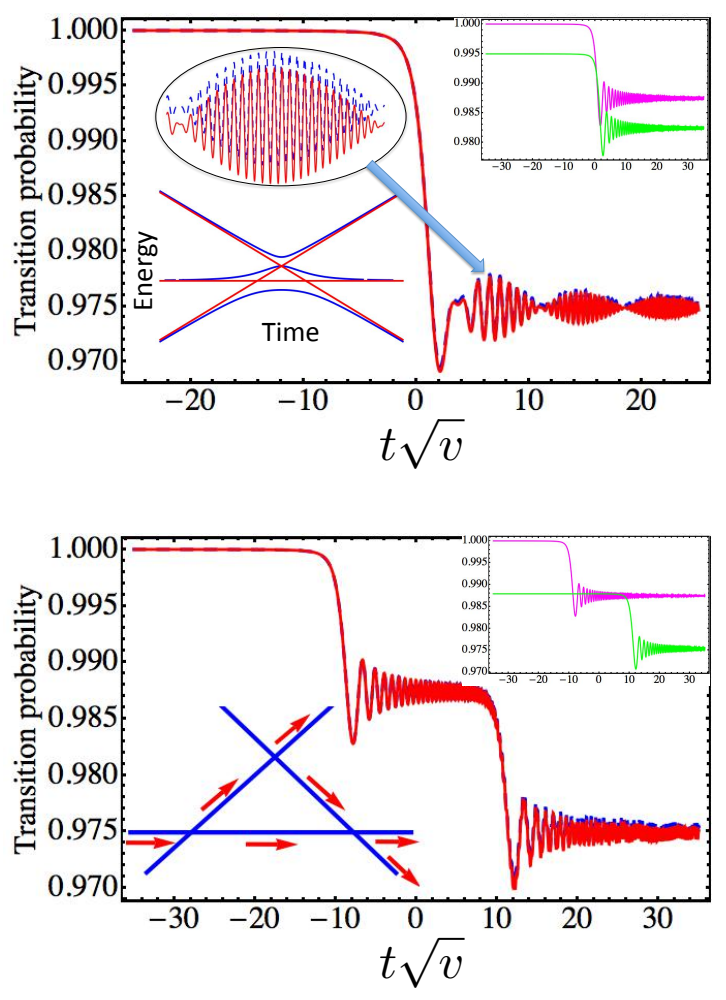

Fig. 2: (Color online) Upper panel: the "beats" calculated at $\Delta^{2} / v=0.004, D / \sqrt{v}=0.425$. Insert 1 : structure of diabatic (red) and adiabatic (blue) states. Insert 2: Zoomed in part of the plot indicated by a blue arrow. Insert 3: Two level crossing probabilities ignoring the interference term. The probability through the first crossing (magenta) at $t_{0}=0$ is used as the initial condition for the transition through the second crossing (green). Lower panel: the "steps" calculated at $\Delta^{2} / v=0.004$, $D / \sqrt{v}=10$. Insert 1: two paths for $\mathrm{SU}(3)$ interferometer built out of three singlet states of triple quantum dot. Insert 2: Two level crossing probabilities ignoring the interference term. The asymptotic value of transition probability through first crossing (magenta) is used as initial condition for the second crossing (green). For both plots dashed blue line stands for numerical solution of the Schrödinger equation for diabatic probability $P_{22}$ in the limit $\delta=\Delta^{2} / v \ll 1$. Solid red line denotes approx(6) imate analytic solution of the Bloch equation 8 15 for $P_{22}$ (16) subject to initial conditions $Q(-\infty)=0, R(-\infty)=-2$ and $W(-\infty)=0$. The solution is valid for arbitrary $\eta=D^{2} / v$. The period of "beats" $T_{\text {beats }} \sim 1 / D$, the size of "steps" plateau is $T_{\text {steps }} \sim D / v$ (see discussion in the text).

triangle work as two splitters while the right vertex performs mixing. We discuss as an example the transmission probability to remain in the same (middle) diabatic state (denoted by $P_{22}$ ). One possibility to arrive at this state is to come along the middle diabatic state. Another one is to go through the upper vertex of the triangle responsible also for a "leakage" from the interferometer. The condition whether we get "beats" or "steps" should depend on a dwell time in the interferometer. The existence of this new pattern is fully attributed to $\mathrm{SU}(3)$ symmetry where the dynamics of the middle adiabatic state is non-trivial (see 
the insert 1 in the upper panel of Fig. 2), being contrasted to trivial dynamics for the symmetric bow-tie model where the diabatic and adiabatic states are the same.

In order to construct a consistent analytic description of the SU(3) LZ transition we analyse the equation for the density matrix (DM) in the non-adiabatic limit. The DM can be parametrized by the set of Gell-Mann matrices

$$
\hat{\rho}(t)=\frac{1}{3} \hat{\mathbb{I}}+\frac{1}{\sqrt{3}} \hat{\vec{\lambda}} \cdot \vec{n}(t)
$$

here $\vec{n}$ is a unit vector in 8-dimensional space of $\mathrm{SU}(3)$ generators. Following a standard procedure we derive a system of Bloch (von Neumann) equations

$$
\frac{d}{d t} \vec{n}=\vec{B}(t) \times \vec{n}(t)
$$

where the cross-product is defined as $(\vec{B} \times \vec{n})_{\alpha}=$ $f_{\alpha \beta \gamma} B_{\beta} n_{\gamma}$ and $f_{\alpha \beta \gamma}$ are totally antisymmetric under exchange of any two indices structure constants of $\mathrm{SU}(3)$ group defined by commutation relations $\left[\lambda_{\alpha}, \lambda_{\beta}\right]=$ $2 i f_{\alpha \beta \gamma} \lambda_{\gamma}$

$$
f_{\alpha \beta \gamma}=\frac{1}{4 i} \operatorname{Tr}\left(\left[\lambda_{\alpha}, \lambda_{\beta}\right] \cdot \lambda_{\gamma}\right)
$$

These equations describe non-dissipative dynamics of the unit vector on a Bloch surface.

In the conventional Gell-Mann basis the generic Hamiltonian $\mathrm{H}_{3}$ describing TTQD (Fig.1a) with all three nonzero tunnel matrix elements between dots contains five $\lambda$ matrices,

$$
\begin{aligned}
\vec{B}^{T} & =\{v t+D, \sqrt{3} v t-D / \sqrt{3}, \Delta \sqrt{2}, \Delta \sqrt{2}, \Delta \sqrt{2}\}, \\
\vec{\Lambda}^{T} & =\frac{1}{2}\left\{\lambda_{3}, \lambda_{8}, \lambda_{1}, \lambda_{4}, \lambda_{6}\right\}
\end{aligned}
$$

Here, the time-dependent level positions $E_{1,2,3}(t)$ are associated with the matrices $\lambda_{3}, \lambda_{8}$ and inter-level transitions are represented by the matrices $\lambda_{1}, \lambda_{4}, \lambda_{6}$.

The SU(3) LZ Hamiltonian (5) may be also rewritten in terms of the differences between the energy levels (1), by means of appropriate combination of the Gell-Mann matrices 25]. Two of three differences, e.g., $E_{10}=\epsilon(t)+U$ and $E_{0 \overline{1}}=\epsilon(t)-U$ may be chosen. In this case the effective field $\vec{B}$ and the spinor $\vec{\Lambda}$ are

$$
\begin{aligned}
\vec{B}^{T} & =\{2(v t+U) / 3,2(v t-U) / 3, \Delta \sqrt{2}, \Delta \sqrt{2}, \Delta \sqrt{2}\} \\
\vec{\Lambda}^{T} & =\frac{1}{2}\left\{\lambda_{3}, \lambda_{-}, \lambda_{1}, \lambda_{4}, \lambda_{6}\right\}
\end{aligned}
$$

with $\lambda_{ \pm}=\left( \pm \lambda_{3}+\sqrt{3} \lambda_{8}\right) / 2$.

To minimize the number of components in the model Hamiltonian, we consider the case of LTQD with suppressed transition $|1\rangle \rightarrow|\overline{1}\rangle$, so that the matrix $\lambda_{4}$ is excluded from $\mathrm{H}_{3}$. This model is straightforwardly mapped on the $\mathrm{S}=1$ Hamiltonian with easy axis, and one may use

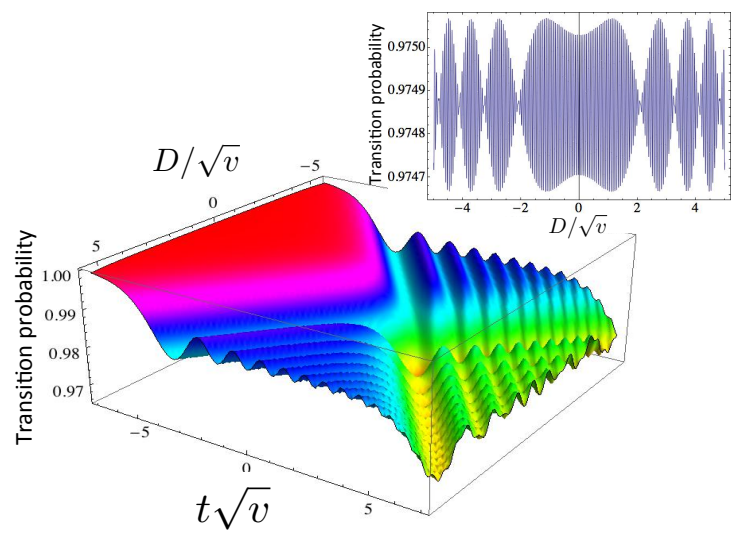

Fig. 3: (Color online) Non-adiabatic transition probability $P_{22}$ as a function of dimensionless time $t / \tau$ and dimensionless uniaxis anisotropy $D \tau$ (LZ time $\tau=1 / \sqrt{v}$ ) computed at $\delta=$ 0.004 . The symmetry $D \rightarrow-D$ reflects the symmetry between "easy axis" and "easy plane" anisotropy of zero-dimensional system. Insert: Transition probability $P_{22}$ at $t \sqrt{v}=100$ as a function of dimensionless dwell time $\sqrt{v} t_{D}=D / \sqrt{v}$ computed at $\delta=0.004$ by Eq16.

a rotated $\mu$-basis of Gell-Mann matrices by applying a unitary transformation to the $\lambda$-basis

$$
\begin{aligned}
& \mu_{1}=\left(\lambda_{1}+\lambda_{6}\right) / \sqrt{2}, \quad \mu_{2}=\left(\lambda_{2}+\lambda_{7}\right) / \sqrt{2} \\
& \mu_{3}=\left(\sqrt{3} \lambda_{8}+\lambda_{3}\right) / 2, \quad \mu_{4}=\lambda_{4} \\
& \mu_{5}=\lambda_{5}, \quad \mu_{6}=-\left(\lambda_{1}-\lambda_{6}\right) / \sqrt{2} \\
& \mu_{7}=-\left(\lambda_{2}-\lambda_{7}\right) / \sqrt{2}, \quad \mu_{8}=\left(\lambda_{8}-\sqrt{3} \lambda_{3}\right) / 2 .
\end{aligned}
$$

The first three $\mu$-matrices coincide with the $\mathrm{SU}(2)$ generators of $S=1$ representation. All commutation notations and Casimir operators are preserved. The two-parametric family of Landau-Zener Hamiltonians corresponds to onedirectional $\mathrm{SU}(3)$ deformation of $\mathrm{SU}(2) \mathrm{LZ}$ model. The "magnetic field" vector and the spinor $\vec{\Lambda}$ in this case are

$$
\vec{B}^{T}=\{\Delta, v t,-D / \sqrt{3}\}, \quad \vec{\Lambda}^{T}=\left\{\mu_{1}, \mu_{3}, \mu_{8}\right\} .
$$

In this representations all interlevel transitions are associated with $\mu_{1}$, the time-dependent level splitting $\epsilon(t)$ is related to $\mu_{3}$ and the matrix $\mu_{8}$ is coupled to the anisotropy parameter.

The transition probabilities (diagonal elements of the density matrix) depend only on $Q$ and $R: \rho_{11}=$ $\frac{1}{3}\left(1+\frac{R}{2}+\frac{3 Q}{2}\right), \rho_{22}=\frac{1}{3}(1-R) \rho_{33}=\frac{1}{3}\left(1+\frac{R}{2}-\frac{3 Q}{2}\right)$. The initial conditions for the lower state occupied at $t=-\infty$ reads: $Q(-\infty)=R(-\infty)=1, W(-\infty)=0$. The initial condition for middle state occupied at $t=-\infty$ is $Q(-\infty)=0, R(-\infty)=-2, W(-\infty)=0$. The initial conditions for upper state occupied reads: $-Q(-\infty)=$ $R(-\infty)=1, W(-\infty)=0$. We also notice a symmetry $\rho_{11} \rightarrow \rho_{33}$ when $Q \rightarrow-Q$ which can be used for mapping of LZ transitions with first and third initial conditions [34]. 
The eight coupled linear differential equations (8) can be transferred into a system of three coupled linear integral equations as follows:

$$
\begin{aligned}
Q(t) & =Q(-\infty)-\frac{\Delta^{2}}{2} \int_{-\infty}^{t} d t_{1} \int_{-\infty}^{t_{1}} d t_{2}\left(K_{r}^{+}\left(t_{1}, t_{2}\right) Q\left(t_{2}\right)+K_{r}^{-}\left(t_{1}, t_{2}\right) R\left(t_{2}\right)\right)+\frac{\Delta}{2} \int_{-\infty}^{t} d t_{1} \Phi_{-}\left(t_{1}\right), \\
R(t) & =R(-\infty)-\frac{3 \Delta^{2}}{2} \int_{-\infty}^{t} d t_{1} \int_{-\infty}^{t_{1}} d t_{2}\left(K_{r}^{+}\left(t_{1}, t_{2}\right) R\left(t_{2}\right)+K_{r}^{-}\left(t_{1}, t_{2}\right) Q\left(t_{2}\right)\right)+\frac{3 \Delta}{2} \int_{-\infty}^{t} d t_{1} \Phi_{+}\left(t_{1}\right), \\
W(t) & =W(-\infty)+\Delta \int_{-\infty}^{t} d t_{1}\left(K_{i}^{+}\left(t, t_{1}\right) R\left(t_{1}\right)+K_{i}^{-}\left(t, t_{1}\right) Q\left(t_{1}\right)\right)+\Phi_{0}(t),
\end{aligned}
$$

where

$$
\begin{aligned}
\Phi_{ \pm}(t) & =-\frac{\Delta}{3} \int_{-\infty}^{t} d t_{1} \int_{-\infty}^{t_{1}} d t_{2}\left(\left[K_{r}^{20} \cdot K_{r}^{ \pm}-K_{i}^{20} \cdot K_{i}^{ \pm}\right] \frac{d}{d t_{2}} R\left(t_{2}\right)+\frac{3 \Delta}{2}\left[K_{r}^{20} \cdot K_{i}^{ \pm}+K_{i}^{20} \cdot K_{r}^{ \pm}\right] W\left(t_{2}\right)\right), \\
\Phi_{0}(t) & =\frac{\Delta}{3} \int_{-\infty}^{t} d t_{1} \int_{-\infty}^{t_{1}} d t_{2}\left(\left[K_{r}^{20} \cdot K_{i}^{+}+K_{i}^{20} \cdot K_{r}^{+}\right] \frac{d}{d t_{2}} R\left(t_{2}\right)-\frac{3 \Delta}{2}\left[K_{r}^{20} \cdot K_{r}^{+}-K_{i}^{20} \cdot K_{i}^{+}\right] W\left(t_{2}\right)\right) .
\end{aligned}
$$

Here we used the notations $K_{r}^{\xi}=\operatorname{Re} \exp \left(i\left(\xi(t)-\xi\left(t_{1}\right)\right), K_{i}^{\xi}=\operatorname{Im} \exp \left(i\left(\xi(t)-\xi\left(t_{1}\right)\right), K_{r / i}^{ \pm}=K_{r / i}^{\Omega^{+}} \pm K_{r / i}^{\Omega^{-}}\right.\right.$, $K_{r / i}^{20}=K_{r / i}^{2 \Omega_{0}}$, and $\Omega_{0}=v t^{2} / 2, \Omega^{ \pm}=\frac{v}{2}\left(t \pm \frac{D}{v}\right)^{2}-\frac{\eta}{2}$. The product $K_{\alpha}^{\beta} \cdot K_{\gamma}^{\delta}=K_{\alpha}^{\beta}\left(t_{1}, t_{2}\right) K_{\gamma}^{\delta}\left(t, t_{1}\right)$. The fact that only three real functions are sufficient for complete parametrization of the DM has very simple explanation. Let's take the limit $D \rightarrow \infty$. In that case we consider three independent $\mathrm{SU}(2) s=1 / 2 \mathrm{LZ}$ transitions accounting also that the tunnel matrix element in the upper vertex of triangle scales as $\Delta^{2} / D[33$. Since the Bloch equations for each of two-level crossings are the equations for transition probabilities conserved in each vertex separately, we have just 3 real functions to describe this limit. Since the $\mathrm{SU}(2)$ limit $D=0$ is also parametrized by 3 real functions [27, the upper and lower bound for the number of functions coincide and is equal to 3 . This reduction is related to some hidden dynamical symmetry connected with higher Casimir invariant of SU(3) group [30].

5. Results and discussion. The system of equations can be solved by iterations in the non-adiabatic limit. The analytic solution for diabatic probability $P_{22}$ in the limit $\delta=\Delta^{2} / v \ll 1$ is given by

$P_{22}(t) \approx 1-\pi \delta\left[F\left(t-\frac{D}{v}\right)+F\left(t+\frac{D}{v}\right)\right]+O\left(\delta^{2}\right)$

where

$F(t)=\frac{1}{2}\left[\left(\frac{1}{2}+C\left(\sqrt{\frac{v}{\pi}} t\right)\right)^{2}+\left(\frac{1}{2}+S\left(\sqrt{\frac{v}{\pi}} t\right)\right)^{2}\right]$

and $C(z)$ and $S(z)$ are cosine and sine Fresnel integrals respectively. The exact solution can be obtained by exponentiation of the first order expression with correction function calculated by means of the method elaborated in 27. The equation (16) shows two "waves": one comes from the first splitting at $t_{-}=-D / v$ and another one comes from the second splitter $/$ mixer at $t_{+}=D / v$. If the period of non-adiabatic oscillations $\tau \sim 1 / \sqrt{v} 36$ is large compared to a dwell time $t_{D} \sim D / v$, which is proportional to linear geometric size of the triangle, the two waves interfere constructively forming the "beats" pattern (Fig.2 upper panel). In that case

$F\left(t-t_{+}\right)+F\left(t-t_{-}\right)-2 F(t) \sim \sin (\pi D t) G\left(\sqrt{\frac{v}{\pi}} t\right)$ where $G(z)=\cos \left(z^{2}\right)(S(z)+1 / 2)-\sin \left(z^{2}\right)(C(z)+1 / 2)$. The period of "beats" is therefore $T_{\text {beats }} \sim 1 / D$. Comparison of these results with the probabilities for two independent two-level crossings without interference term (Insert 3 in Fig.2, upper panel) unambiguously demonstrates the key role of the interference processes. If, however, the period of non-adiabatic oscillations is small compared to the dwell time $\tau<t_{D}$, the double splitting of initial state (two consequent LZ transitions, see the Insert 2 of the Fig. 2, lower panel) leads to formation of the "steps" pattern (Fig.2. lower panel). The characteristic time for the "steps" (the size of a plateau) is the dwell time $T_{\text {steps }} \sim D / v$. One can see that there are pronounced non-adiabatic oscillations in the plateau of the steps. In order to see both "beats" and "steps" the system should be prepared in any pure state (we showed in Fig.2 and Fig. 3 the results corresponding to initial conditions given by occupied middle diabatic state) and the state $S_{101}$ should be used as a probe for the interference pattern. The analytic solution of the Bloch equation (14), (15) shown by solid red curve demonstrates remarkable agreement with corresponding numerical solution of the Schrödinger equation (blue dashed curve). The approach based on solution of Bloch equations allows one to consider effects of classical fast and slow noise 27 by either ensemble averaging the equation [35] if the noise is fast or averaging its solution in given realization if the 
noise is slow 27. The noise is associated with fluctuations of the Overhauser field (double- and triple- quantum dots experiments), fluctuations of electric field (Immanuel Bloch experiments) and fluctuations of both charge and flux in superconducting qubits. Besides, the Bloch equation approach allows treatment of periodically driven LZ systems involving mixed quantum mechanical states. We leave these problems for future studies 30 . The results of SU(3) LZ interference are summarized in Fig.3. The pattern shows the oscillations due to the two-path interference in the non-adiabatic limit. Transition probability at long times $t \gg t_{D}$ also shows pronounced "beats" structure characteristic for two-path interference (see insert of Fig.3). The equations (8)-(15), the "beats" and "steps" shown in Fig. 2 and the interference pattern (Fig.3) represent the central results of this Letter.

Conclusions. We analysed general models of 3-level crossing in the space of SU(3) generators (5) or pseudospin 1 with anisotropy (2)-(4) and presented both numerical solution of the Schrödinger equation and approximate analytical solution of the Bloch (von Neumann) equation. Excellent agreement between two approaches is demonstrated. If the diabatic states of linearly driven 3-level system form a triangle, it acts as an interferometer with qualitatively new pattern of interference oscillations. Depending on the dwell time in the triangle, the interference pattern shows the "beats" due to constructive interference of two paths or "steps" when two separated in time non-adiabatic LZ transitions take place. Both "beats" and "steps" are the manifestations of SU(3) symmetry. We believe that the interference pattern predicted in this work can be experimentally observed both in quantum transport (TQD) and in ultra-cold bosons experiments.

We are grateful to B.L. Altshuler, G. Burkard, A. Chudnovskiy, Yu. Galperin, V. Gritsev, S. Ludwig, G. Mussardo, J.Petta and L. Vandersypen for illuminating discussions. The work of MBK is supported by STEP program at ICTP. MNK appreciates the hospitality at LMU (Munich) where part of this work has been done. The research of MNK was supported in part by the National Science Foundation under Grant No. NSF PHY11-25915.

\section{REFERENCES}

[1] L. D. Landau, Phys. Z. Sowietunion 2, 46 (1932).

[2] C. Zener, Proc. R. Soc. A. 1371, 696 (1932).

[3] E. C. G. Stückelberg, Helv. Phys. Acta 5, 369 (1932).

[4] E. Majorana, Nuovo Cimento 9, 43 (1932).

[5] H. Nakamura, Non-adiabatic transitions (World Scientific, Singapur 2002).

[6] Yu. Nazarov and Ya. Blanter. Quantum Transport: Introduction to Nanoscience (Cambridge University Press, Cambridge 2009).

[7] David D. Awschalom. Spin electronics. (Kluwer Academic, Dordrecht 2004).

[8] W. Wernsdorfer and R. Sessoli, Science 284, 133 (1999).

[9] S. Trotzky et al, Phys. Rev. Lett. 105, 265303 (2010); C. Kasztelan et al, Phys. Rev. Lett. 106, 155302 (2011); M.
Aidelsburger et al, Phys. Rev. Lett 107, 255301 (2011); S. Trotzky et al, Nat. Phys. 8, 325 (2012).

[10] P. Cheinet et al, Phys. Rev. Lett. 101, 090404 (2008).

[11] D. Loss, D.P. DiVincenzo, Phys. Rev. A 57, 120(1998)

[12] R. Hanson at al, LRev. Mod. Phys. 79, 12171265 (2007)

[13] S. Shevchenko, S. Ashhab, F. Nori. Phys. Rep. 492, 1 (2010)

[14] E. Paladino et al, arXiv: 1304.7925 to be published in Rev. Mod. Phys.

[15] Y. Nakamura, C.D. Chen, J.S.Tsai, Phys. Rev. Lett. 79, 2328 (1997).

[16] J.E. Mooji et al, Science 285, 1036 (1999)

[17] T. Hayashi et al, Phys. Rev. Lett. 91, 226804 (2003).

[18] J.R. Petta et al, Phys. Rev. Lett. 93, 186802 (2004); J.R. Petta et al, Science 30921802184 (2005); J.M. Taylor et al, Nat. Phys. 1, 177 (2005); S. Foletti et al, Nat. Phys. 5, 903 (2009); H. Bluhm et al, Phys. Rev. Lett. 105, 216803 (2010); H. Bluhm et al, Nat. Phys. 7, 109 (2011).

[19] Experimental realization of electronic equivalent of optical Mach-Zehnder- interferometer in DQD has been discussed in: J. R. Petta, H. Lu, A. C. Gossard. Science 327, 669 (2010)

[20] C.M. Quintana et al, Phys. Rev. Lett. 110, 173603 (2013).

[21] D.M. Schröer et al, Phys. Rev. B 76, 075306 (2007).

[22] H. Ribeiro, J.R. Petta, G. Burkard. arXiv:1210.1957

[23] F.R. Braakman et al, arXiv:1303.1034 arXiv:1303.2533

[24] S. Amaha et al, Phys. Lett. 94, 092103 (2009).

[25] K. Kikoin, M. N. Kiselev and Y. Avishai: Dynamical Symmetry for Nanostructures. Implicit Symmetry in SingleElectron Transport Through Real and Artificial Molecules. (Springer. Wien-New York, 2012).

[26] C. E. Carroll and F. T. Hioe, J. Phys. A 19, 1151 (1986); C. E. Carroll and F. T. Hioe, J. Phys. A 19, 2061 (1986); S. Brundobler and V. Elser, J. Phys. A 26, 1211 (1993); V. N. Ostrovsky and H. Nakamura, J. Phys. A 30, 6939, (1997); Y. N. Demkov and V. N. Ostrovsky, Phys. Rev. A 56, 032705, (2000); Y. N. Demkov and V. N. Ostrovsky, J. Phys. B 34, 2419, (2001).

[27] M.B. Kenmoe et al, Phys. Rev. B 87, 224301 (2013).

[28] G. S. Vasilev, S. S. Ivanov, and N. V. Vitanov, Phys. Rev. A 75, 013417, (2007).

[29] The Hamiltonians $H_{1}-H_{3}$ can be derived microscopically both for quantum transport and cold-gases setups. See details in 25].

[30] M.B. Kenmoe, M.N. Kiselev, K.A.Kikoin (unpublished)

[31] H. Georgi, Lie Algebras in Particle Physics. 2nd Ed., (Westview, 1999).

[32] Only non-zero components of 8-vector are shown. The constant term $H_{0}=-\frac{2 D}{3} \hat{\mathbb{I}}$ is omitted from the Hamiltonian to shift the middle diabatic state to zero energy.

[33] All three gaps between adiabatic states scale as $\Delta$ in the limit $D \ll \Delta$.

[34] In the $\mathrm{SU}(2)$ limit $D=0$ and $\Phi_{-}=K^{-}=0$. The equation for $Q$ represents the vector $\mathrm{SU}(2) S=1$ Bloch equation being decoupled from the second and third equations which form a tensor Bloch equation 35.

[35] V. L. Pokrovsky and N. A. Sinitsyn, Phys. Rev. B 67, 144303 (2003), V. L. Pokrovsky and N. A. Sinitsyn, Phys. Rev. B 69, 104414 (2004).

[36] K. Mullen et al, Phys. Rev. Lett. 62, 2543 (1989). 